\title{
Intercalated combination of chemotherapy and erlotinib for stage IIIA non-small-cell lung cancer: a multicenter, open-label, single-arm, phase II study
}

This article was published in the following Dove Press journal: Cancer Management and Research

\author{
Zhiwei Chen $^{1, *}$ \\ Shengping Shen ${ }^{1, *}$ \\ Wenbo Shi ${ }^{1}$,* \\ Gening Jiang ${ }^{2}$ \\ Xin Wang ${ }^{3}$ \\ Hong Jian' \\ Zhen Zhou' \\ Zhengping Ding' \\ Shun Lu' \\ 'Tumor Clinical Medical Center, Shanghai \\ Chest Hospital, Shanghai Jiao Tong \\ University, Shanghai 200030, People's \\ Republic of China; ${ }^{2}$ Department of \\ Thoracic Surgery, Shanghai Pulmonary \\ Hospital, Tongji University, Shanghai \\ 200433, People's Republic of China; \\ ${ }^{3}$ Department of Thoracic Surgery, Sun \\ Yat-Sen University Cancer Center, \\ Guangzhou, People's Republic of China
}

*These authors contributed equally to this work
Correspondence: Zhengping Ding; Shun Lu Tumor Clinical Medical Center, Shanghai Chest Hospital, Shanghai Jiao Tong University, 24I Huaihai West Road, Shanghai 200030, People's Republic of China

Tel +862 I22 200000 Ext 2I5I

Email lushun_1970@163.com;

t4m972@163.com
Objective: This multicenter, open-label, single-arm, phase II trial evaluated the efficacy and safety of an intercalated combination of erlotinib and gemcitabine/cisplatin or carboplatin in patients with stage IIIA non-small-cell lung cancer (NSCLC).

Registration: This trial is registered with ClinicalTrials.gov, number NCT01297101.

Methods: The primary endpoint was the objective response rate (ORR), which includes complete response (CR) and partial response (PR), assessed using RECIST version 1.0 in the intention-to-treat population. Adverse events (AEs) were graded by the Common Terminology Criteria for Adverse Events (CTCAE) version 3.0. Secondary endpoints included the disease control rate, disease-free survival (DFS), overall survival (OS), and safety. Between April 1, 2011, and July 31, 2014, 39 patients with stage IIIA NSCLC received two cycles of intercalated use of erlotinib with gemcitabine/cisplatin or carboplatin. Results: Eighteen patients $(46.15 \%)$ achieved a PR and no patient achieved a pathologic CR, resulting in an ORR of $46.15 \%$ (95\% CI 30-63\%). Median DFS was 20 months (95\% CI 5.26-50.61) and median OS was 25 months (95\% CI 15.57-33.39). Patients with EGFR mutations $(n=7)$ had a higher ORR than those with wild-type EGFR $(n=9)(85.71 \%$ vs $55.56 \%, \mathrm{P}=0.00$ ). Most AEs were CTCAE grade 1 or 2 ; there were no cases of increased hematologic toxicity or erlotinib-emergent interstitial lung disease observed.

Conclusion: Two cycles of intercalated neoadjuvant therapy with erlotinib and gemcitabine/ cisplatin or carboplatin were effective and safe for patients with stage IIIA NSCLC. This approach should be further explored in larger randomized controlled trials given the lack of a consensus about the best treatment for stage IIIA NSCLC.

Keywords: non-small-cell lung cancer, NSCLC, neoadjuvant, erlotinib, gemcitabine, platinum, objective response rate, progression-free survival, overall survival

\section{Introduction}

Lung cancer accounts for $17 \%$ of new cancer cases and approximately one-quarter of all cancer deaths globally. ${ }^{1}$ In China, it is estimated that there were 490 patients newly diagnosed with lung cancer per 1 million people in 2005 and that the mortality rate has increased 1.46 -fold over the past 7 years. $^{2}$ Non-small-cell lung cancer (NSCLC) is the most common type of lung cancer and is traditionally managed with surgical resection, radiotherapy, and chemotherapy. Curative-intent surgery remains the preferred treatment modality for NSCLC, but its efficacy is limited because patients typically present with unresectable locally advanced or metastatic tumors. ${ }^{3}$ In these cases, platinum-based chemotherapy with regimens 
like gemcitabine plus carboplatin has long been regarded as standard first-line therapy, ${ }^{4,5}$ with median progressionfree survival (PFS) of 4 to 6 months. ${ }^{6}$

Identification of gene mutations in NSCLC, including epidermal growth factor receptor (EGFR) mutations, has led to molecularly stratified therapy of NSCLC with regimens like the EGFR tyrosine kinase inhibitors (EGFRTKI). The IPASS Study demonstrated that EGFR-TKI therapy was superior to conventional chemotherapy in terms of both PFS and the objective response rate (ORR) in patients with lung cancer who harbored EGFR-sensitive mutations. ${ }^{7}$ Erlotinib is a highly potent reversible EGFRTKI. However, erlotinib with concurrent cisplatin and gemcitabine has been shown to confer no survival benefit compared with chemotherapy alone in chemotherapynaïve advanced NSCLC patients. ${ }^{8}$ In the TRIBUTE trial, ${ }^{9}$ erlotinib plus standard first-line chemotherapy failed to improve survival in treatment-naïve stage IIIB/IV NSCLC patients. The lack of overall benefit in these studies raises questions about concurrent therapy with erlotinib and chemotherapeutic agents and suggests that alternative regimens are needed. The FASTACT and FASTACT-2 studies showed that intercalated use of erlotinib and chemotherapy significantly improved PFS in previously untreated stage IIIB/IV NSCLC patients; ${ }^{10}$ however, no difference was observed in OS. Our previous study ${ }^{11}$ using an intercalated gefitinib regimen showed significant improvement in PFS and OS in selected patients with stage IIIB/IV non-squamous NSCLC. A recent systemic review and meta-analysis of 10 randomized controlled trials (six with erlotinib and four with gefitinib) further confirmed the improvements in PFS and OS with intercalated combination therapy. ${ }^{12}$

Stage IIIA NSCLC patients represent a heterogeneous group, and their management remains a clinical challenge. ${ }^{13}$ A recent study showed that $85 \%$ of stage IIIA NSCLC patients received chemoradiotherapy alone, with the remaining $15 \%$ receiving chemoradiotherapy and surgery. ${ }^{14}$ Surgical resection alone does not extend survival in stage IIIA NSCLC patients, who have a 5year overall survival (OS) of only $15 \%$. This is partially attributed to the fact that by the time of diagnosis, micro-metastasis is present in $80 \%$ of stage IIIA NSCLC patients, ${ }^{15}$ making complete tumor (R0) resection a challenge. Clinical studies have indicated that a multimodality approach with neoadjuvant therapy followed by surgery improves the survival of stage IIIA NSCLC patients. ${ }^{16-18}$
Currently, there are no data on neoadjuvant chemotherapy intercalated with erlotinib in stage IIIA NSCLC patients. In this multicenter, open-label, single-arm, phase II trial, we investigated the efficacy and safety of intercalated use of erlotinib with neoadjuvant therapy with gemcitabine/cisplatin or carboplatin in stage IIIA NSCLC patients.

\section{Patients and methods}

\section{Patients}

This study was conducted at Shanghai Chest Hospital, Shanghai Jiao Tong University, Shanghai Pulmonary Hospital, Tongji University, and Sun Yat-sen University Cancer Center, Guangzhou, China, between April 1, 2011, and July 31, 2014. It enrolled patients with histologically or cytologically proven and CT-confirmed American Joint Committee on Cancer (AJCC) stage IIIA NSCLC. Patients were eligible if 1 ) they were $\geq 18$ years of age; 2 ) they had an Eastern Cooperative Oncology Group (ECOG) performance status of 0 or $1 ; 3$ ) they had a life expectancy of $\geq 12$ weeks; and 4) they had adequate information on bone marrow, hematologic, renal, and liver function (required laboratory tests: neutrophil count, hemoglobin, platelets, serum total bilirubin, aspartate aminotransferase, alanine aminotransferase, calculated creatinine clearance, prothrombin, and activated partial thromboplastin time). Patients were also required to have at least one measurable lesion at baseline according to the Response Evaluation Criteria in Solid Tumors (RECIST) version 1.0. Those who had received prior systemic anti-cancer therapy, including cytotoxic therapy, erlotinib, other EGFR TKIs, antibodies targeting EGFR, or any other experimental therapies, were excluded. Other major exclusion criteria were known allergy to erlotinib or any other drugs used in the study, locoregional radiotherapy for NSCLC, interstitial pneumonitis, a history of cancer other than NSCLC within the preceding 5 years (except cervical carcinoma in situ, cured basal cell carcinoma, and bladder epithelial tumors), severe cardiac disease, severe systemic disease, lack of compliance or cooperation, ocular inflammation or infection that was not fully under control, and major surgery or severe trauma within the preceding 2 months. Pregnant or lactating women were also excluded.

The study protocol was approved by the ethics committee of each participating center and the study was performed in accordance with the Declaration of Helsinki and Good Clinical Practice guidelines. All patients 
provided written informed consent before participation in the study. This trial was registered at ClinicalTrials.gov (NCT01297101).

\section{Study intervention}

All patients received intravenous gemcitabine $\left(1000 \mathrm{mg} / \mathrm{m}^{2}\right)$ on days 1 and 8 plus cisplatin $\left(75 \mathrm{mg} / \mathrm{m}^{2}\right)$ or carboplatin $(A U C=5)$ on day 1 and oral erlotinib $(150 \mathrm{mg} /$ day $)$ intercalated on days 15 to 28 . Each cycle consisted of 28 days. The study medications were continued for two cycles or until disease progression, unacceptable toxicity, or withdrawal of consent. Modifications or delays in administration of the study drugs were based on the worst toxicity grade according to NCI CTCAE version 3.0. Dose modifications of gemcitabine or carboplatin were based on the nadir of the absolute neutrophil or thrombocyte counts of the previous cycle. When administration of the study medication was delayed for $\geq 3$ weeks or was discontinued due to toxicity or because a reduction of three dose levels was required, the patient was withdrawn from the study.

\section{Patient evaluation}

Data on patient demographics, including age, gender, medical history including smoking history, and concurrent therapy, were collected. All patients were evaluated monthly with a physical examination that included ECOG performance status, routine laboratory investigations, and electrocardiography. A chest and abdominal computed tomography (CT) scan was performed at baseline, at the end of neoadjuvant therapy, and when clinical disease progression was suspected. The ORR was the proportion of patients in the intention-to-treat (ITT) population who achieved a complete response (CR) or partial response (PR) assessed using the Response Evaluation Criteria in Solid Tumors (RECIST) version 1.0. The disease control rate (DCR) was the percentage of patients who achieved a CR or PR or stable disease (SD) in the ITT population. Pathologic CR (pCR) was defined as the complete disappearance of tumor cells in all pathological specimens after surgical resection in patients who had received neoadjuvant therapy. Patients who achieved remission and were eligible for surgery underwent curative surgical resection. The curative surgical resection rate was the percentage of patients in the ITT population who underwent curative surgical resection.

Patients were followed up at 3-month intervals for 2 years from the completion of neoadjuvant therapy or until disease recurrence or death. Disease-free survival (DFS) was calculated from the day of surgical resection to the first day of recurrence, death from any cause, or the last day of follow-up. OS was calculated from the day when therapy was initiated to the last day of follow-up or death from any cause.

\section{Safety}

Adverse events (AEs) were graded using the CTCAE version 3.0. Vital signs and AEs were monitored throughout the study. Safety assessments were based mainly on the occurrence, frequency, and severity of AEs. For all AEs, when necessary, patients were withdrawn from the study. Safety analysis included all patients who received at least one dose of the study drug and had at least one follow-up safety assessment. Safety assessments were analyzed mainly using descriptive statistics.

\section{EGFR mutation analysis}

Blood samples and surgically resected fresh tumor specimens were obtained. Tumor DNA was extracted using the QIAamp DNA FFPE tissue kit (Qiagen, Crawley, UK) and pyrosequencing was performed to detect all 43 reported EGFR mutations. The Sanger method was used to sequence 28 exons of the EGFR gene.

\section{Statistical analysis}

It was estimated that a sample size of 36 patients would be needed to achieve a reported ORR of $43 \%$ (95\% CI $33.7-63.3 \%, \alpha=0.05, \beta=0.2)$ with an assumed two-sided type I error of 0.05 and a dropout rate of $10 \%{ }^{7}$ Statistical analyses were carried out using the SAS software package, version 9.4 (SAS Institute Inc., Cary, NC). Statistical analyses were prespecified before the database was locked and followed the ITT principle. The ITT population included all patients who received at least one dose of the study drug and had a baseline assessment and at least one post-baseline assessment. The primary endpoint was the ORR, and secondary endpoints included the disease control rate, DFS, OS, and safety. Median DFS and OS were estimated with the Kaplan-Meier method and compared by log-rank test. The treatment effects were expressed as hazard ratios (HRs) with two-sided 95\% confidence intervals (CI) and estimated using the Cox proportional hazards model. The ORR of the ITT population was analyzed using Fisher's exact test. DFS and OS durations were censored at the last day of follow-up for 
patients who were still alive or whose disease had not progressed.

All tests were two-tailed with a level of significance set at $\alpha=0.05$. All statistical analyses were done using the SAS 9.3 software package.

\section{Results}

\section{Patient demographic and baseline characteristics}

Thirty-nine patients with stage IIIA NSCLC were enrolled in the study. All patients received at least one dose of the study drug and had a baseline assessment and at least one post-baseline assessment and were included in the ITT population. Patient demographic and baseline characteristics are shown in Table 1. Most patients were male (74.36\%), were current or former smokers $(61.54 \%)$, had good

Table I Patient demographic and baseline characteristics

\begin{tabular}{|l|l|}
\hline Characteristic & Patients (n=39) \\
\hline Age, years & \\
Median & 59 \\
Range & 52,63 \\
\hline Female gender, n(\%) & $10(25.64)$ \\
\hline Smoking status, n(\%) & \\
Smokers & \\
Ever former smokers^ & $14(35.90)$ \\
Never smokers* & $10(25.64)$ \\
\hline Pathology, n(\%) & $15(38.46)$ \\
Adenocarcinoma & \\
Adenosquamous carcinoma & $18(46.15)$ \\
Others & $13(33.33)$ \\
\hline EGFR mutation status, n(\%) & $8(20.51)$ \\
Positive & \\
Exon 19 & $7(17.95)$ \\
Exon 20 & 5 \\
Exon 19/2I & 1 \\
Negative & 1 \\
Unknown & $9(23.08)$ \\
\hline Brain CT or MRI abnormality, n(\%) & $23(58.97)$ \\
Yes & $19(48.72)$ \\
No & $18(46.15)$ \\
Unknown & $2(5.13)$ \\
\hline
\end{tabular}

Notes: ${ }^{\$}$ Smokers are defined as current smokers smoking $>100$ cigarettes/lifetime, or smoking >100 cigarettes/lifetime with abstinence from smoking for less than I year on the day before the start of therapy. ${ }^{\wedge}$ Ever former smokers are defined as those who had abstained from smoking for at least I year on the day before the start of therapy and had a history of at least 100 cigarettes/lifetime. ${ }^{*}$ Never smokers are defined as those smoking $<100$ cigarettes/lifetime. performance status (ECOG 0 or 1), and had adenocarcinoma $(46.15 \%)$ or adenosquamous carcinoma $(33.33 \%)$.

Seven patients (17.95\%) had confirmed EGFR mutations, and nine patients (23.08\%) had wild-type EGFR. The remaining 23 patients (58.97\%) had unknown EGFR mutation status.

\section{Treatment characteristics}

All 39 patients completed two cycles of neoadjuvant therapy with gemcitabine plus carboplatin or cisplatin with intercalated erlotinib. The mean total dose of gemcitabine for the cohort was $6755.74 \pm 796.29 \mathrm{mg} / \mathrm{m}^{2}$ (median: $6800.00 \mathrm{mg} / \mathrm{m}^{2}$; range: 3200.00 to $8000.00 \mathrm{mg} / \mathrm{m}^{2}$ ). Twenty-six patients received carboplatin and 13 patients received cisplatin. The mean total dose of carboplatin was $1162.76 \pm 201.03 \mathrm{mg} / \mathrm{m}^{2}$ (median: $1260.00 \mathrm{mg} / \mathrm{m}^{2}$; range: 645.00 to $1500.00 \mathrm{mg} / \mathrm{m}^{2}$ ) and that of cisplatin was $256.15 \pm 13.13 \mathrm{mg} / \mathrm{m}^{2}$ (median: $257.00 \mathrm{mg} / \mathrm{m}^{2}$; range: 240.00 to $280.00 \mathrm{mg} / \mathrm{m}^{2}$ ). The mean total dose of erlotinib was $3992.11 \pm 623.43 \mathrm{mg}$ (median: $4200 \mathrm{mg}$; range: 1200 to $4200 \mathrm{mg})$. Twenty-two patients $(56.41 \%)$ underwent surgical resection, including 15 patients who received curative surgical resection and seven who received non-curative surgical resection. The curative resection rate was $68.2 \%$.

\section{Efficacy}

Eighteen patients (46.15\%) achieved a PR and 18 (46.15\%) had SD. No patient achieved a pCR. The ORR was $46.15 \%(95 \%$ CI $30-63 \%)$ and the DCR was $92 \%$ (95\% CI 79-98\%). Univariate analysis showed that gender, age ( $\leq 60 v s>60$ years), smoking status, adenocarcinoma ( $v s$ other types), EGFR mutational status, and ECOG scores were not significant determinants of the ORR (Table S1). Additionally, a waterfall plot of the maximum tumor shrinkage from baseline is shown in Figure 1. The mean sum of the longest diameters of measurable lesions in the study cohort was $82.62 \pm 32.84 \mathrm{~cm}$ (median: $80 \mathrm{~cm}$; interquartile range [IQR]: 55 to $105 \mathrm{~cm}$ ) at baseline and $63.45 \pm 33.68 \mathrm{~cm}$ (median: $57 \mathrm{~cm}$; IQR: 38 to $87 \mathrm{~cm}$ ) at the final follow-up visit, a significant $23.92 \%$ reduction over baseline $(\mathrm{P}<0.0001)$.

Median DFS was 20 months (95\% CI, 5.3-50.6) (Figure 2A). The Kaplan-Meier DFS curve is shown in Figure 2B for patients stratified by EGFR mutational status. Cox proportional hazards analysis showed no statistically significant difference in DFS for patients harboring wild-type $E G F R$ versus those harboring mutated $E G F R$ (HR 2.60; 95\% CI 0.683-9.907; $\mathrm{P}=1.1612$ ) or those with 


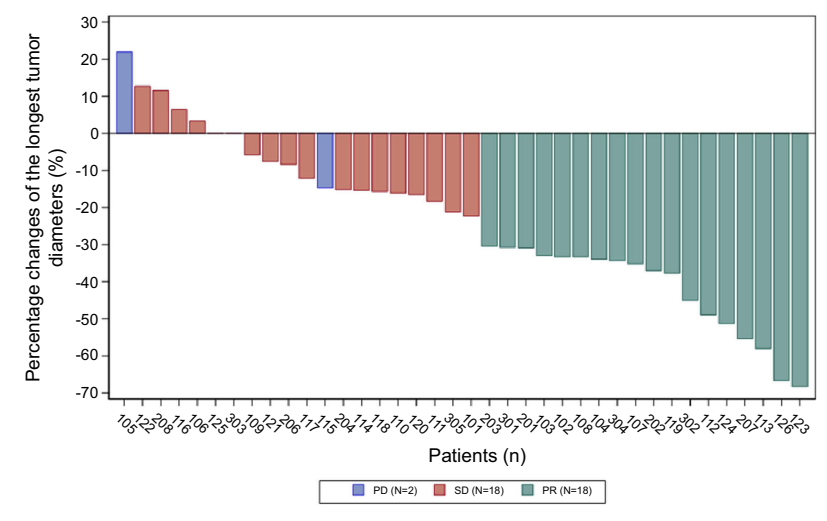

Figure I Waterfall plot of the largest percentage changes from baseline in the sum of the longest tumor diameters for stage IIIA NSCLC patients.

Abbreviations: CR, complete response; NSCLC, non-small-cell lung cancer; PD, progressive disease; PR, partial response; $S D$, stable disease. unknown EGFR mutational status (HR 1.598; 95\% CI $0.344-7.421 ; \mathrm{P}=0.5496)$. Among patients undergoing surgical resection, those with wild-type EGFR had a longer median DFS (24 months; 95\% CI, 1.22-50.61) than those with mutated EGFR (5 months), but the difference was not statistically significant $(\mathrm{P}=0.352)$ (Table 2 ).

Median OS for the entire cohort was 25 months (95\% CI 15.57-33.39) (Figure 3A), but it was higher for patients who underwent surgical resection compared with those who did not (33 vs 17 months, log-rank test $\mathrm{P}=0.0076$ ) (Figure 3B). Cox proportional hazards analysis showed that surgical resection conferred a significantly reduced mortality risk versus no surgical resection (HR 0.33 ; 95\% CI 0.14-0.78). Patients with wild-type EGFR had a longer median OS

\section{A}
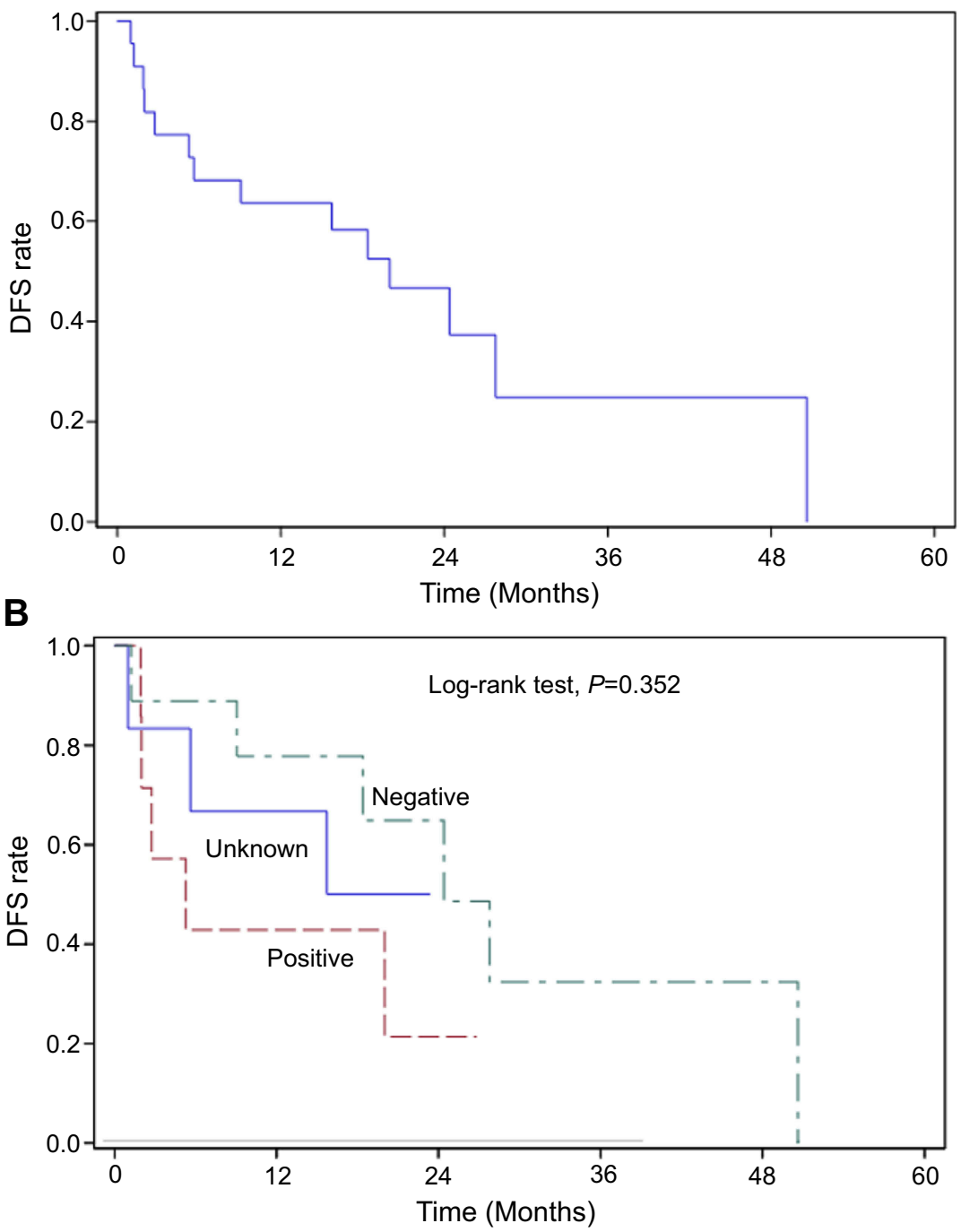

Figure 2 Kaplan-Meier curves for (A) disease-free survival (DFS) of the intention-to-treat population and (B) DFS of the intention-to-treat population stratified by EGFR mutational status.

Abbreviation: $\mathrm{Cl}$, confidence interval. 
Table 2 Median DFS of stage IIIA NSCLC patients undergoing surgical resection, stratified by EGFR mutational status ${ }^{\&}$

\begin{tabular}{|l|l|l|l|l|l|}
\hline & Wild-type EGFR & Mutated EGFR & Unknown EGFR status & Total (n=22) & P-value^ \\
\hline Events (censored) & $6(3)$ & $5(2)$ & $3(3)$ & $14(8)$ & \\
\hline Median DFS, months (95\% Cl) & $24(1.22,50.61)$ & $5(1.92, *)$ & $*(0.99, *)$ & $20(5.26,50.61)$ & 0.3520 \\
\hline
\end{tabular}

Notes: ${ }^{\&}$ calculated by the Kaplan-Meier method. ^Log-rank test for comparison of the three groups.

Abbreviations: DFS, disease free survival; NSCLC, non-small-cell lung cancer.

(44 months) than those with mutated EGFR (26 months) and those with unknown EGFR mutational status (18 months) (Figure 3C). Cox proportional hazards analysis showed that mutated EGFR was associated with an increased mortality risk versus wild-type EGFR (HR 2.26; 95\% CI $0.437-$ 11.673; $\mathrm{P}=0.3311$ ) and unknown EGFR mutational status (HR 3.75; 95\% CI 1.077-13.062; $\mathrm{P}=0.0379$ ). Among patients undergoing surgical resection, mutated EGFR was associated with a numerically but nonsignificantly higher mortality risk versus wild-type EGFR (HR 2.26; 95\% CI 0.399-12.818; $\mathrm{P}=0.3568$ ). Similarly, unknown EGFR mutational status was associated with a numerically but nonsignificantly higher mortality risk versus wild-type EGFR (HR 1.76; 95\% CI 0.350-8.869; $\mathrm{P}=0.4918$ ) (Figure 3D).
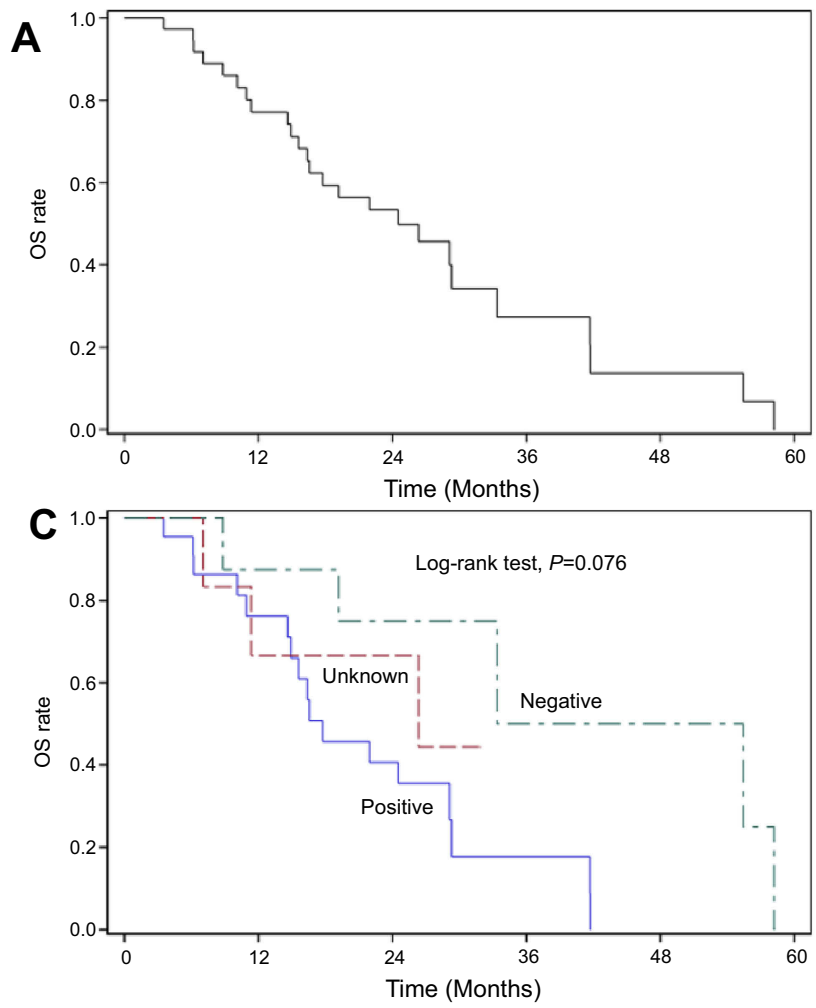

\section{Safety}

Safety data are summarized in Table 3. Most AEs were CTCAE grade 1 or 2 and only $12.8 \%$ of AEs were CTCAE $\geq$ grade 3 . No AEs were CTCAE grade 4 . Furthermore, $69.2 \%$ patients experienced a treatmentemergent AE. The most common AEs (occurring in $\geq 10 \%$; all grades) were leucopenia (38.5\%), rash (28.2\%), elevated alanine aminotransferase $(20.5 \%)$, neutropenia $(20.5 \%)$, thrombocytopenia $(12.8 \%)$, and vomiting (10.3\%). Moreover, $17.9 \%$ patients experienced treatment-emergent AEs that led to treatment interruption or dose reduction. No cases of increased hematologic toxicity or erlotinib-emergent interstitial lung disease were observed.
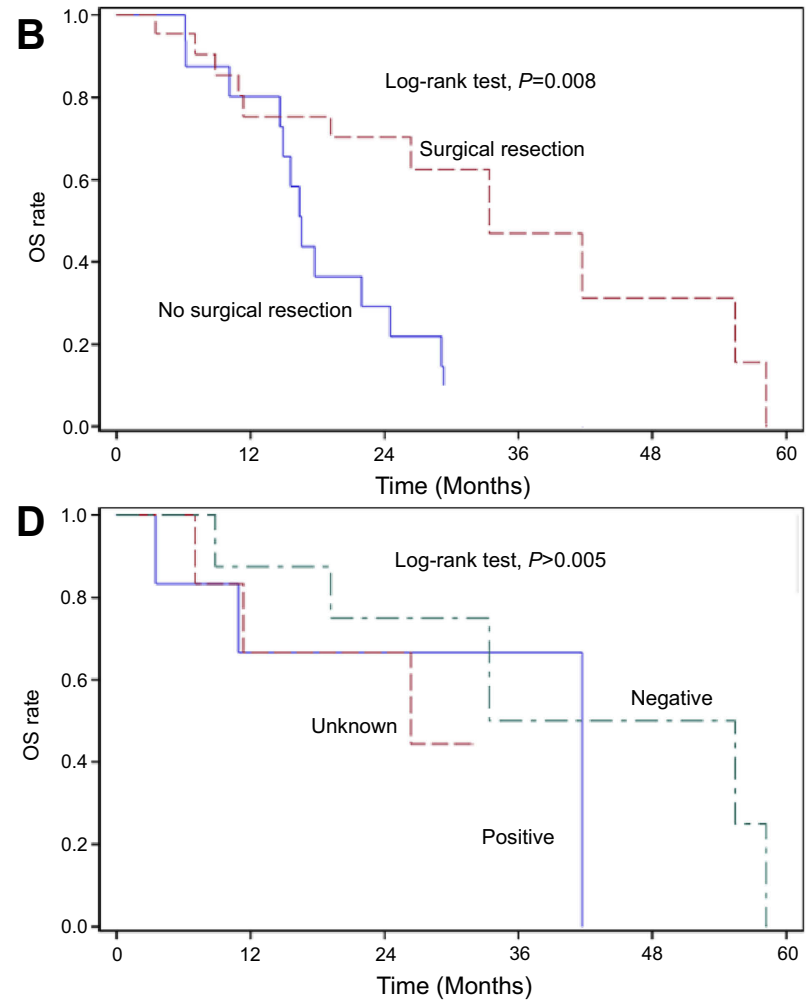

Figure 3 Kaplan-Meier curves for (A) overall survival (OS) of the intention-to-treat population, (B) OS of the intention-to-treat population stratified by surgical resection versus no surgical resection, (C) OS of the intention-to-treat population stratified by EGFR mutational status, and (D) OS of surgical patients stratified by EGFR mutational status. Abbreviation: $\mathrm{Cl}$, confidence interval. 
Table 3 Adverse events (all grades >10\%); $n(\%)$

\begin{tabular}{|c|c|c|}
\hline AEs (all grades $>10 \%$ ) & No. of events & Cases, $\mathrm{n}(\%)$ \\
\hline Total AEs & 99 & $27(69.2)$ \\
\hline \multicolumn{3}{|l|}{ CTCAE Grade (3.0) } \\
\hline I & 55 & $20(51.3)$ \\
\hline 2 & 39 & $18(46.2)$ \\
\hline 3 & 5 & $5(12.8)$ \\
\hline 4 & 0 & 0 \\
\hline $\begin{array}{l}\text { AEs leading to interruption } \\
\text { or dose reductions }\end{array}$ & 11 & $7(17.9)$ \\
\hline ADR & 94 & $27(69.2)$ \\
\hline SAE & 0 & 0 \\
\hline AEs CTCAE grade $\geq 3$ & 5 & $5(12.8)$ \\
\hline Death & 0 & 0 \\
\hline
\end{tabular}

Abbreviations: ADR, adverse drug reaction; AEs, adverse events; CTCAE, CommonTerminology Criteria for Adverse Events; SAE, severe adverse event.

\section{Discussion}

Intercalated erlotinib therapy with chemotherapy improves PFS and OS in previously untreated patients with stage IIIB/IV NSCLC ${ }^{10,19}$ or selected advanced NSCLC patients who have achieved SD with chemotherapy. ${ }^{11}$ The current open-label, single-arm, phase II trial demonstrated that intercalated use of erlotinib with neoadjuvant therapy with gemcitabine/cisplatin or carboplatin resulted in an ORR of $46.15 \%$ (95\% CI $30-63 \%)$ and a median OS of 25 months $(95 \%$ CI $15.57-33.39)$ in patients with stage IIIA NSCLC.

Stage IIIA NSCLC patients are a heterogeneous group with diverse presentations ranging from apparently resectable tumors with occult microscopic nodal metastases to unresectable multistation nodal disease. ${ }^{20}$ Currently, there is lack of consensus regarding the optimal treatment of various stage IIIA subsets, mainly due to a lack of meaningful, large randomized trials. ${ }^{13,21}$ Large randomized trials on stage IIIB/ IV NSCLC patients showed no significant survival benefit of erlotinib with concurrent cisplatin and gemcitabine versus chemotherapy alone, ${ }^{8,9,22}$ while intercalated use of erlotinib with neoadjuvant therapy led to significant improvement in PFS and OS. ${ }^{10,19}$ The intercalated regimen used in the current study on stage IIIA NSCLC patients yielded an ORR of $46.15 \%$ (95\% CI 30-63\%), which is numerically higher than the ORR of $41.7 \%$ reported by Zhong et al for EGFRmutated stage IIIA-N2 NSCLC patients receiving neoadjuvant therapy with erlotinib alone. ${ }^{23}$
Our study cohort included various subsets of stage IIIA NSCLC patients, including IIIA-N2 NSCLC. Only $17.95 \%$ of patients had confirmed EGFR mutations, $23.08 \%$ had wild-type EGFR, and the rest (58.97\%) had unknown EGFR mutation status. The ORR for EGFRmutated patients was $85.7 \%$, higher than that reported by Zhong et al, ${ }^{23}$ suggesting that the efficacy of the intercalated use of erlotinib with gemcitabine and platinum-based drugs is superior to that of neoadjuvant therapy with erlotinib alone. Approximately one-third of stage IIIA NSCLC patients have unresectable tumors; slightly more than half of our patients were operable (56.41\%). We observed that operable patients had significantly longer DFS and OS versus inoperable patients, with Cox proportional hazards analysis showing that surgical resectability was a significant determinant of survival for stage IIIA NSCLC patients.

Biomarker analysis of the phase III TORCH trial evaluating first-line erlotinib versus chemotherapy in unselected advanced NSCLC patients showed that EGFR mutation was a significant predictor of PFS. ${ }^{24}$ Accumulating evidence indicates that NSCLC patients with activating EGFR mutations derive significant clinical benefits from EGFR-TKI therapy. EGFR mutational status is unknown in approximately one-fifth of NSCLC patients in Western countries ${ }^{25,26}$ and an even greater proportion in less developed countries. ${ }^{27}$ One limitation of the current study is the low rate of EGFRmutation testing (41.03\%). Our subgroup analysis showed that patients with mutated EGFR had a numerically higher, though statistically nonsignificant, ORR (85.71\%) compared with those with wild-type EGFR (55.56\%), suggesting that stage IIIA NSCLC patients with activating EGFR mutations may derive greater clinical benefits from intercalated neoadjuvant therapy with EGFR-TKI and with gemcitabine/cisplatin or carboplatin. This finding should be confirmed in future studies given the small size of the current cohort and the low rate of EGFR-mutation testing.

Our study showed that intercalated use of erlotinib with gemcitabine/cisplatin or carboplatin was safe for stage IIIA NSCLC patients. Most AEs were CTCAE grade 1 or 2 and the most common AEs (occurring in $\geq 10 \%$; all grades) were leucopenia and skin rash. No cases of increased hematologic toxicity or erlotinib-emergent interstitial lung disease or death were observed. Wu et $\mathrm{al}^{19}$ observed more toxicity related to EGFR-TKI, including skin rash and diarrhea, and more treatment-emergent death in the chemotherapy plus erlotinib group than in the chemotherapy plus placebo group. Our study used a regimen similar to that used by $\mathrm{Wu}$ 
et al except that intercalated neoadjuvant therapy was given for two rather than six cycles.

The study has several major limitations. It is a singlearm study that lacks a control arm. Furthermore, the sample size is rather small, with only 39 patients. Therefore, our findings need to be confirmed in future prospective randomized controlled studies with larger patient populations. In addition, patients were not selected for the current study by their EGFR-mutational status, and slightly more than half of the cohort had unknown EGFR mutation status. The study was initiated in 2013 at a time when EGFR testing was not widely adopted in China and other Asian countries; several factors affected use of EGFR testing, including inadequate specimen size, lack of testing facilities or equipment, and patient reluctance to undergo testing because of financial concerns. ${ }^{27,28}$ Though EGFR testing has become more common in Asia and other parts of the world, still not all NSCLC patients receive EGFR testing; therefore, the findings of the current study are of relevance for unselected NSCLC patients.

\section{Conclusion}

Our open-label, single-arm, phase II trial demonstrated that two cycles of intercalated neoadjuvant therapy with erlotinib and gemcitabine/cisplatin or carboplatin were effective and safe for stage IIIA NSCLC. This approach should be further explored in larger randomized controlled trials given the lack of a consensus about the best treatment for stage IIIA NSCLC.

\section{Ethics approval and informed consent}

The study protocol was approved by the ethics committee of each participating center and the study was performed in accordance with the Declaration of Helsinki and Good Clinical Practice guidelines. All patients provided written informed consent before participation in the study. The trial was registered at ClinicalTrials.gov (NCT01297101) on February 16, 2011.

\section{Availability of data and materials}

The datasets supporting the conclusions of this article are included within the article.

\section{Funding}

Our publication fee was paid for by EDANZ GROUP JAPAN K.K, a Japanese supplier of Shanghai Roche
Pharmaceutical Co., Ltd, and this research is sponsored by Roche.

\section{Disclosure}

The authors report no conflicts of interest in regard to this work.

\section{References}

1. Jemal A, Bray F, Center MM, Ferlay J, Ward E, Forman D. Global cancer statistics. CA Cancer J Clin. 2011;61(2):69-90. doi:10.3322/ caac. 20107

2. Chen W, Sun K, Zheng R, et al. Cancer incidence and mortality in China, 2014. Chin J Cancer Res. 2018;30(1):1-12. doi:10.21147/j. issn.1000-9604.2018.01.01

3. Rapp E, Pater JL, Willan A, et al. Chemotherapy can prolong survival in patients with advanced non-small-cell lung cancer-report of a Canadian multicenter randomized trial. J Clin Oncol. 1988;6 (4):633-641. doi:10.1200/JCO.1988.6.4.633

4. Pfister DG, Johnson DH, Azzoli CG, et al. American Society of Clinical Oncology treatment of unresectable non-small-cell lung cancer guideline: update 2003. J Clin Oncol. 2004;22(2):330-353. doi:10.1200/JCO.2004.09.053

5. Schiller JH, Harrington D, Belani CP, et al. Comparison of four chemotherapy regimens for advanced non-small-cell lung cancer. $N$ Engl J Med. 2002;346(2):92-98. doi:10.1056/NEJMoa011954

6. Azzoli CG, Baker S, Temin S, et al. American Society of Clinical Oncology Clinical Practice Guideline update on chemotherapy for stage IV non-small-cell lung cancer. J Clin Oncol. 2009;27 (36):6251-6266. doi:10.1200/JCO.2009.23.5622

7. Mok TS, Wu Y-L, Thongprasert S, et al. Gefitinib or carboplatinpaclitaxel in pulmonary adenocarcinoma. $N$ Engl J Med. 2009;361 (10):947-957. doi:10.1056/NEJMoa0810699

8. Gatzemeier U, Pluzanska A, Szczesna A, et al. Phase III study of erlotinib in combination with cisplatin and gemcitabine in advanced non-small-cell lung cancer: the Tarceva lung cancer investigation trial. J Clin Oncol. 2007;25(12):1545-1552. doi:10.1200/ JCO.2005.05.1474

9. Herbst RS, Prager D, Hermann R, et al. TRIBUTE: a phase III trial of erlotinib hydrochloride (OSI-774) combined with carboplatin and paclitaxel chemotherapy in advanced non-small-cell lung cancer. J Clin Oncol. 2005;23(25):5892-5899. doi:10.1200/JCO.2005.02. 840

10. Mok TSK, Wu Y-L, Yu C-J, et al. Randomized, placebo-controlled, phase II study of sequential erlotinib and chemotherapy as first-line treatment for advanced non-small-cell lung cancer. J Clin Oncol. 2009;27(30):5080-5087. doi:10.1200/JCO.2008.21.5541

11. Jian H, Li W, Ma Z, et al. Intercalating and maintenance gefitinib plus chemotherapy versus chemotherapy alone in selected advanced non-small cell lung cancer with unknown EGFR status. Sci Rep. 2017;7(1):8483. doi:10.1038/s41598-017-08399-8

12. La Salvia A, Rossi A, Galetta D, et al. Intercalated chemotherapy and epidermal growth factor receptor inhibitors for patients with advanced non-small-cell lung cancer: a systematic review and meta-analysis. Clin Lung Cancer. 2017;18(1):23-33 e1. doi:10.1016/j.cllc.2016.08.006

13. Rocco G, Nason K, Brunelli A, Varela G, Waddell T, Jones DR. Management of stage IIIA (N2) non-small cell lung cancer: a transatlantic perspective. J Thorac Cardiovasc Surg. 2016;151(5):12351238. doi:10.1016/j.jtcvs.2016.01.035

14. Patel AP, Crabtree TD, Bell JM, et al. National patterns of care and outcomes after combined modality therapy for stage IIIA non-smallcell lung cancer. J Thorac Oncol. 2014;9(5):612-621. doi:10.1097/ JTO.0000000000000152 
15. Bunn PA Jr. Future directions in clinical research for lung cancer. Chest. 1994;106(6 Suppl):399S-407S. doi:10.1378/chest. 106.6_supplement.399s

16. Group NM-AC. Preoperative chemotherapy for non-small-cell lung cancer: a systematic review and meta-analysis of individual participant data Lancet. 2014;383(9928):1561-1571. doi:10.1016/S0140-6736(13)62159-5

17. Rosell R, Gómez-Codina J, Camps C, et al. A randomized trial comparing preoperative chemotherapy plus surgery with surgery alone in patients with non-small-cell lung cancer. $N$ Engl $J$ Med. 1994;330(3):153-158. doi:10.1056/NEJM199401203300301

18. Roth JA, Fossella F, Komaki R, et al. A randomized trial comparing perioperative chemotherapy and surgery with surgery alone in resectable stage IIIA non-small-cell lung cancer. J Natl Cancer Inst. 1994;86(9):673-680. doi:10.1093/jnci/86.9.673

19. Wu Y-L, Lee JS, Thongprasert $\mathrm{S}$, et al. Intercalated combination of chemotherapy and erlotinib for patients with advanced stage non-smallcell lung cancer (FASTACT-2): a randomised, double-blind trial. Lancet Oncol. 2013;14(8):777-786. doi:10.1016/S1470-2045(13)70254-7

20. Ramnath N, Dilling TJ, Harris LJ, et al. Treatment of stage III non-small cell lung cancer: diagnosis and management of lung cancer, 3rd ed: American College of Chest Physicians evidence-based clinical practice guidelines. Chest. 2013;143(5 Suppl):e314S-e340S. doi:10.1378/chest.12-2360

21. Robinson LA, Ruckdeschel JC, Wagner H, Stevens CW. Treatment of non-small cell lung cancer-stage IIIA: ACCP evidence-based clinical practice guidelines (2nd edition). Chest. 2007;132(3 Suppl):243S265S. doi:10.1378/chest.07-1379

22. Pisters KMW, Vallières E, Crowley JJ, et al. Surgery with or without preoperative paclitaxel and carboplatin in early-stage non-smallcell lung cancer: Southwest Oncology Group Trial S9900, an intergroup, randomized, phase III trial. J Clin Oncol. 2010;28(11):18431849. doi: $10.1200 / J C O .2009 .26 .1685$
23. Zhong W, Yang X, Yan H, et al. Phase II study of biomarker-guided neoadjuvant treatment strategy for IIIA-N2 non-small cell lung cancer based on epidermal growth factor receptor mutation status. $J$ Hematol Oncol. 2015;8:54. doi:10.1186/s13045-015-0151-3

24. Kim L, Saieg M, Di Maio M, et al. Biomarker analysis of the phase 3 TORCH trial for first line erlotinib versus chemotherapy in advanced non-small cell lung cancer patients. Oncotarget. 2017;8(34):5752857536. doi:10.18632/oncotarget. 15725

25. Douillard J-Y, Ostoros G, Cobo M, et al. Gefitinib treatment in EGFR mutated caucasian NSCLC: circulating-free tumor DNA as a surrogate for determination of EGFR status. J Thorac Oncol. 2014;9 (9):1345-1353. doi:10.1097/JTO.0000000000000263

26. Kimura H, Suminoe M, Kasahara K, et al. Evaluation of epidermal growth factor receptor mutation status in serum DNA as a predictor of response to gefitinib (IRESSA). Br J Cancer. 2007;97(6):778-784. doi:10.1038/sj.bjc.6603949

27. Salto-Tellez M, Tsao M-S, Shih J-Y, et al. Clinical and testing protocols for the analysis of epidermal growth factor receptor mutations in East Asian patients with non-small cell lung cancer: a combined clinical-molecular pathological approach. J Thorac Oncol. 2011;6 (10):1663-1669. doi:10.1097/JTO.0b01-3e318227816a

28. Yatabe Y, Kerr KM, Utomo A, et al. EGFR mutation testing practices within the Asia Pacific region: results of a multicenter diagnostic survey. J Thorac Oncol. 2015;10(3):438-445. doi:10.1097/ JTO.0000000000000422 


\section{Supplementary material}

Table SI Univariate analysis of determinants of the objective response rate in the intention-to-treat population

\begin{tabular}{|c|c|c|c|}
\hline Variables & $\mathbf{N}(\%)$ & $95 \% \mathrm{Cl}^{*}$ & $\mathbf{P}$ \\
\hline \multicolumn{4}{|l|}{ Gender } \\
\hline Male & $12(4 \mid .38)$ & $0.47 \mathrm{I}(0.101,2.004)$ & 0.3133 \\
\hline Female & $6(60.00)$ & & \\
\hline \multicolumn{4}{|l|}{ Smoking status, n(\%) } \\
\hline Current smokers & $4(28.57)$ & $0.400(0.068,2.150)$ & 0.2900 \\
\hline Ever former smokers & $5(50.00)$ & & \\
\hline Never smokers & $9(60.00)$ & I.500 (0.296,7.83I) & 0.6224 \\
\hline \multicolumn{4}{|l|}{ Histopathology } \\
\hline Adenocarcinoma & $8(44.44)$ & $0.880(0.244,3.130)$ & 0.8430 \\
\hline Others & $10(47.62)$ & & \\
\hline \multicolumn{4}{|l|}{ Age, years } \\
\hline$>60$ & $7(50.00)$ & $1.273(0.339,4.817)$ & 0.7186 \\
\hline$\leq 60$ & II (44.00) & & \\
\hline \multicolumn{4}{|l|}{ EGFR mutation } \\
\hline Yes & $6(85.7 I)$ & $4.798(0.497,111.595)$ & 0.2174 \\
\hline No & $5(55.56)$ & & \\
\hline Unknown & $7(30.43)$ & $0.350(0.067,1.697)$ & 0.1947 \\
\hline \multicolumn{4}{|l|}{ ECOG } \\
\hline I & $9(45.00)$ & $0.909(0.255,3.230)$ & 0.8821 \\
\hline 0 & $9(47.37)$ & & \\
\hline
\end{tabular}

Abbreviations: $\mathrm{Cl}$, confidence interval; $\mathrm{N}$, number

\section{Publish your work in this journal}

Cancer Management and Research is an international, peer-reviewed open access journal focusing on cancer research and the optimal use of preventative and integrated treatment interventions to achieve improved outcomes, enhanced survival and quality of life for the cancer patient.
The manuscript management system is completely online and includes a very quick and fair peer-review system, which is all easy to use Visit http://www.dovepress.com/testimonials.php to read real quotes from published authors. 